\title{
Impaired responses of leukemic dendritic cells derived from a human myeloid cell line to LPS stimulation
}

\author{
Kwang Dong Kim², Seung-Chul Choi ${ }^{2}$, \\ Young-Woock $\mathrm{Noh}^{2}$, Jong Wan $\mathrm{Kim}^{3}$, \\ Sang-Gi Paik ${ }^{4}$, Young Yang ${ }^{1}$, \\ Keun II $\mathrm{Kim}^{1}$ and Jong-Seok Lim ${ }^{1,5}$ \\ 1Department of Biological Sciences \\ Sookmyung Women's University \\ Seoul 140-742, Korea \\ ${ }^{2}$ Laboratory of Cell Biology \\ Korea Research Institute of Bioscience and Biotechnology \\ Daejeon 305-600, Korea \\ ${ }^{3}$ Laboratory of Medicine \\ Dankook University College of Medicine \\ Cheonan 330-714, Korea \\ ${ }^{4}$ Department of Biology \\ Chungnam National University \\ Daejeon 305-764, Korea \\ ${ }^{5}$ Corresponding author: Tel, 82-2-710-9560; \\ Fax, 82-2-2077-7322; E-mail, jslim@sookmyung.ac.kr
}

Accepted 1 February 2006

Abbreviations: DC, dendritic cells; MoDC, monocyte-derived DC; MuDC, MUTZ-3-derived dendritic cells; rh, recombinant human; TLR, Toll-like receptor

\begin{abstract}
Several myeloid leukemia-derived cells have been reported to possess the ability to differentiate into dendritic cells (DC). MUTZ-3, a myeloid leukemia cell line, responds to GM-CSF, IL-4 and TNF- $\alpha$, and acquires a phenotype similar to immature monocyte-derived DC (MoDC). In the present study, MUTZ-3-derived DC (MuDC) showed high level expression of HLA class II molecules, CD80 and CD86, and were able to function as potent antigen presenting cells as previously reported. Interes tingly, MuDC maturation was induced by CD40mediated stimulation, but not by LPS stimulation. We analyzed CCR1, CCR7 and Toll-like receptor (TLR) expressions in MuDC, and measured IL-10 and IL-12 production after maturation stimuli. Although MuDC expressed the mRNA for TLR4, a major component of the LPS receptor system, they did not show an enhanced level of CCR7 or cytokine production after LPS stimulation. In contrast, they responded to CD40
\end{abstract}

stimulation, which resulted in increased levels of CD83, CD86 and CCR7. Moreover, while LPSstimulated MoDC could potently stimulate NK cells in a DC-NK cell co-culture, LPS-stimulated MuDC failed to stimulate primary NK cells. Taken together, our findings suggest that, although MuDC express TLR4, unlike TNF- $\alpha$ and IL-1 $\beta$, LPS does not stimulate MuDC to acquire mature phenotypes, and they may have impaired activity to initiate innate immune response.

Keywords: antigens, CD40; cell differentiation; dendritic cells; killer cells, natural; lipopolysaccharides; Toll-like receptors

\section{Introduction}

Dendritic cells (DC) are professional APC, derived from hematopoietic progenitor cells, and play a central role in the initiation of the immune response to pathogens (Banchereau et al., 2000). APC express pattern recognition molecules that are thought to recognize foreign ligands during the early phases of immune responses (Rescigno and Borrow, 2001). The best known of these are probably the Toll-like receptors (TLRs).

Eleven TLR have been identified in mammals up to date and the agonists for most of them have been characterized (Re and Strominger, 2004; Zhang et al., 2004). All are type I integral membrane receptors with extracellular leucine rich regions and intracellular portions that are homologous to the signaling domain of IL-1R. Gene knockout studies in mice indicate that TLR4 is required for LPS responsiveness (Poltorak et al., 1998; Hoshino et al., 1999). The extracellular domain of human TLR4 associates with a second protein, MD2, which is required for optimal LPS-induced signaling (Shimazu et al., 1999). The intracellular domain of TLR4 associates with myeloid differentiation 88 (MyD88), which is an adaptor protein containing a TIR (Toll/interleukin-1 receptor) domain (Fitzgerald et al., 2001). Another adaptor, TIRAP (also known as MAL), especially associates with TLR4, and thus may be responsible for MyD88-independent response (Horng et al., 2001). Human TLR2 and TLR4 recruit and activate IL-1 receptor-associated kinase (IRAK) in response to a variety of pathogen-associated molecular patterns (PAMPs), including the gram-negative bacterial toxin, 
LPS, which results in the downstream activations of $\mathrm{NF}-\kappa \mathrm{B}$ and c-Jun $\mathrm{NH}_{2}$-terminal kinase, and in the secretions of IL-8 and IL-12 (Suzuki et al., 2002).

Much of what is known concerning DC maturation has been learned from primary DC cultures, cells differentiated upon being treated with GM-CSF and IL-4 either from nongrowing human blood monocytes or from proliferating bone marrow-derived precursors. A human cell line exhibiting the characteristics of CD $34^{+}$cell-derived DC precursors would allow the detailed study of DC differentiation without the associated problems of donor variability and DC precursor cell availability. It was recently reported that MUTZ-3, a cell line derived from myeloid leukemia, acquires a cytokine-induced interstitial and immature DC phenotype (Masterson et al., 2002). However, little information is available concerning the responses of primary leukemia- or leukemia cell line-derived DC to maturation-inducing stimuli.

In this study, we demonstrate that MUTZ-3derived DC (MuDC) acquire a cytokine-induced iDC phenotype but they have a defective LPS responsiveness. Unlike LPS response, TNF- $\alpha$-mediated maturation was functional, in terms of the enhancement of costimulatory molecules and the activation of p38 MAPK. In addition, while MoDC could stimulate NK cells in the presence of LPS, MuDC could not enhance NK cell activities. The interaction between MoDC and NK cells plays an important role in the initiation of innate immune response. Thus, our data suggest that myeloid leukemia-derived DC may have impaired activity to initiate innate immune response.

\section{Materials and Methods}

\section{Reagents used for cell culture, antibiotics, and cytokines}

All cultures were performed in RPMI 1640 (Sigma, St. Louis, MO) or $\alpha-M E M$ (Gibco, Grand Island, NY) supplemented with $10 \%$ FBS (Gibco). The cytokines used in the cultures were GM-CSF (LG Biotech, Iksan, South Korea), IL-4 (Pierce Endogen, Woburn, MA), SCF (R\&D Systems, Minneapolis, MN), and TNF- $\alpha$ (PeproTech Inc., Rocky Hill, NJ). The flow cytometric analysis used to determine antigen expression of DC was performed using FITC- or $P E-c o n j u g a t e d$ monoclonal antibodies against the following markers: HLA-DR, CD1a, CD11c, CD14, CD80, CD83, CD86, and CD123 (PharMingen. San Diego, CA). Mouse monoclonal antibody against human TLR4 was purchased from Santa Cruz Biotechnology (Santa Cruz, CA). Goat $F(a b)_{2}$ antimouse IgG-FITC conjugate (Biosource, Camarillo, CA) was used to detect anti-TLR4 antibody. Mouse immunoglobulin $\mathrm{G}_{1}$-FITC/PE isotype control (PharMingen) was used as an isotype control. LPS ( $E$. coli 0127:B8) and IL-1 $\beta$ were purchased from Sigma and R\&D Systems, respectively, and agonistic anti-CD40 antibody (G28.5; HB-9110, ATCC, Rockville, MD) was an affinity-purified form obtained from the ascites of immunized mice.

\section{Generation of MUTZ-3-derived dendritic cells (MuDC) and monocyte-derived dendritic cells (MoDC)}

The cytokine-dependent human cell line MUTZ-3 (Deutsche Sammlung von Mikroorganismen und Zellkulturen, Braunschweig, Germany) was maintained at $5 \times 10^{5} / \mathrm{ml}$ in 12 -well tissue plates (Costar, Cambridge, MA) in $\alpha$-MEM supplemented with SCF $(10 \mathrm{ng} / \mathrm{ml})$. For MuDC generation the cells were cultured at $2 \times 10^{5} / \mathrm{ml}$ in 12 -well tissue plates in the presence of GM-CSF (200 ng/ml), IL-4 (10 ng/ml), and TNF- $\alpha(2.5 \mathrm{ng} / \mathrm{ml})$ for $7 \mathrm{~d}$. For MoDC generation the method described by Sallusto et al. (Sallusto and Lanzavecchia, 1994) was slightly modified. Briefly, PBMC (peripheral blood mononuclear cells) from healthy donors were isolated by density centrifugation on Histopaque 1077 (Sigma), and erythrocytes were then lysed. Cells were harvested and resuspended in RPMI 1640 medium supplemented with L-glutamine $(2 \mathrm{mM})$, penicillin $(100 \mathrm{U} / \mathrm{ml})$, streptomycin $(100 \mu \mathrm{g} / \mathrm{ml})$, HEPES $(10 \mathrm{mM})$, and $10 \%$ heat-inactivated FBS. Plastic adherent cells (2 $\mathrm{h}, 37^{\circ} \mathrm{C}$ ) were cultured overnight, and nonadherent cells were removed. The cells were usually harvested after culturing for 6-7 d, and used for assay. CD $34^{+}$cell-derived DC were generated as previously described (Choi et al., 2003). Briefly, CD34 ${ }^{+}$progenitor cells $\left(2 \times 10^{6}\right.$ cells $\left./ 2 \mathrm{ml}\right)$ isolated from cord blood were cultured in RPMI 1640 containing 10\% FBS in the presence of recombinant human (rh) GM-CSF $(200 \mathrm{ng} / \mathrm{ml})$, rhTNF- $\alpha(2.5 \mathrm{ng} / \mathrm{ml})$ and rhSCF $(20 \mathrm{ng} / \mathrm{ml})$ until day 5 . Afterwards, SCF was replaced by IL-4 $(20 \mathrm{ng} / \mathrm{ml})$ and cells were further cultured for 7-9 d. After 12-14 d of culture, DC were harvested, and used in this study.

\section{Flow cytometric analysis}

DC generated in vitro were subjected to flow cytometric analysis using FACSCalibur and Cell Quest software (Becton Dickinson, Mountain View, $\mathrm{CA})$. The cells were incubated with various conjugated mAbs on ice for $30 \mathrm{~min}$. FITC and PE were simultaneously measured using $530 \mathrm{~nm}$ and $575 \mathrm{~nm}$ filters, in logarithmic mode.

\section{Determination of allogeneic T-cell proliferation}

The allostimulatory capacity of DC that had been 
$\gamma$-irradiated (3000 rad; $\mathrm{Co}^{60}$ ) prior to co-culture was tested in an one-way MLR using allogeneic T lymphocytes $\left(5 \times 10^{4}\right.$ cells/well $)$ at various stimulator/responder cell ratios in triplicates in 96-well U-bottom plates (Corning Glassware, Corning, NY). $\left[{ }^{3} \mathrm{H}\right]-\mathrm{TdR}$ (NEN-DuPont, Boston, MA) was added to each well for the final $16 \mathrm{~h}$ of the 5-day assay. Cell-associated radioactivity was determined by direct $\beta$ counting using a liquid scintillation counter (LS6000LL, Beckman, Palo Alto, CA), and results were expressed as the means of triplicate assays.

\section{Cytokine detection}

IL-10, IL-12, and IFN- $\gamma$ assays were carried out using commercially available cytokine detection kits (Pierce Endogen) according to the manufacturer's instructions. MoDC or MuDC $\left(5 \times 10^{5} / \mathrm{ml}\right)$ were incubated in the presence of LPS or anti-CD40 mAb for $24 \mathrm{~h}$. In other experiments, fresh NK cells and MuDC or MoDC were co-cultured in the absence or presence of LPS for $24 \mathrm{~h}$. Supernatants were collected and the cytokines were quantified using an Emax precision microplate reader (Molecular Devices, Sunnyvale, CA).

\section{Reverse transcriptase (RT)-PCR}

CCR and TLR mRNAs were quantified in MUDC and MoDC by RT-PCR. Total RNAs from MuDC or MoDC were isolated using the guanidinium method. First-strand cDNAs were synthesized using a ProSTAR First-Strand RT-PCR kit (Stratagene, La Jolla, CA). The cDNA was amplified using a PCR premix, Sapphire (Super-Bio, Suwon, South Korea). The predenaturing condition was $5 \mathrm{~min}$ at $94^{\circ} \mathrm{C}$ and an additional $30 \mathrm{~s}$ at $94^{\circ} \mathrm{C}$. Annealing was carried out for $30 \mathrm{~s}$ at $60^{\circ} \mathrm{C}$, followed by $30 \mathrm{~s}$ at $72^{\circ} \mathrm{C}$. Thirty cycles of PCR were performed and this was terminated with a final extension for $10 \mathrm{~min}$ at $72^{\circ} \mathrm{C}$. The sequences of the primers used were as follows: CCR1; sense 5'-TCCTCACGAAAGCCTACGAGAGTGGAAGC-3', antisense 5'-CCACGGAGAGGAAGGGGAGCCATTTAAC-3', CCR7; sense 5'-GATTACATCGGAGACAACAAC-3', antisense 5'-TAGTCCAGGCAGAAGAGTCG-3', TLR1; sense 5'-GGAGAGACTTATGGGGAA-3', antisense 5'-CCAATTGTTGCAGAGACTT-3', TLR2; sense 5'-GGCTGCATATTCCAAGGT-3', antisense 5'-CCGCTCACTGTAAGAAAC-3', TLR3; sense 5'-TCTGAGTAACAGCCAGCT3', antisense 5'- ATTCCTGGCCTGTGAGTT-3', TLR 4; sense 5'-CTGGTGAGTGTGACTATTG-3', antisense 5'-ACCGACACACCAATGATG-3', TLR7; sense 5'-CCCTACTGTTTTGCCATC-3', antisense 5'-TGACAGATTCAGGCATTTG-3', TLR9; sense 5'-ATCGTCAAACTGGCGCCT-3', antisense 5'-TCCGACAGGTCCACGTA-3', TLR10; sense, 5'-GGCAGGTCA-
CAGGTATTT-3', antisense 5'-AGTTCTCGTAAGGCCATC-3'.

\section{Western blotting}

Immature MuDC were washed twice and incubated in serum free medium (Sigma) overnight at $37^{\circ} \mathrm{C}$. Cells were stimulated with LPS $(1 \mu \mathrm{g} / \mathrm{ml})$, TNF- $\alpha$ $(200 \mathrm{ng} / \mathrm{ml})$ or IL-1 $\beta(20 \mathrm{ng} / \mathrm{ml})$, and incubated for indicated times. Cells were harvested and washed once with cold PBS, and pellets were resuspended in RIPA buffer and stood on ice for $1 \mathrm{~h}$. After centrifugation the supernatants were saved. Proteins of $40 \mu \mathrm{g}$ were separated by SDS/PAGE and blotted onto PVDF membranes (Hybond-P. Amersham Pharmacia Biotech, Bucks, UK). Membranes were blocked with $4 \%(\mathrm{w} / \mathrm{v})$ skim milk (Difco Laboratories, Detroit, MI) in PBS for $1 \mathrm{~h}$ at room temperature and then incubated overnight with primary antibody at $4^{\circ} \mathrm{C}$. Antibodies to phospho- and total p38, and phospho- and total ERK were purchased from Cell Signaling Technology (Beverly, MA). Anti-rabbit lgG (H\&L)-HRP was purchased from Cell Signaling Technology. Detection was performed by enhanced chemiluminescence (ECL; Amersham Pharmacia Biotech).

\section{NK-DC co-culture}

Purified allogeneic NK cells were obtained by using a human NK cell isolation kit (Miltenyi Biotec, Bergisch Gladbach, Germany). Resting NK cells expressing CD56 (>95\%) were usually added to MuDC or MoDC at a ratio $1: 1$ and co-cultured in RPMI 1640 medium for $24 \mathrm{~h}$ in the presence or absence of LPS. Prior to co-culture, MuDC or MoDC were removed from their differentiation media by washing with RPMI 1640 medium.

\section{${ }^{51} \mathrm{Cr}$ release assay}

To evaluate the cytolytic activities of NK cells, we used K562 cells as target cells. K562 cell line $(1 \times$ $10^{6}$ ) was incubated with $100 \mu \mathrm{Ci}$ of $\mathrm{Na}_{2}{ }^{51} \mathrm{CrO}_{4}$ (NEN-DuPont) for $60 \mathrm{~min}$ at $37^{\circ} \mathrm{C}$ and then extensively washed. Total $1 \times 10^{4}$ cells in $100 \mu \mathrm{l}$ of complete culture medium were distributed into each well, in triplicate, of a U-bottomed 96-well microtiter plate. After incubation with NK cells for $4 \mathrm{~h}, 100 \mu \mathrm{l}$ of supernatant was collected, and the ${ }^{51} \mathrm{Cr}$ release was counted in a gamma counter (Wallac, Gaithersburg, $M D)$. The specific lysis percentage was calculated as follows: $100 \times[(\mathrm{cpm}$ of test sample $-\mathrm{cpm}$ of spontaneous lysis) / (cpm of maximum control cpm of spontaneous lysis)], where cpm denotes counts per minute. 


\section{Results}

MUTZ-3, in the presence of the cytokine combination, differentiates into DC that have a phenotype similar to monocyte-derived DC

We cultured MUTZ-3 in a medium, which included granulocyte macrophage-colony-stimulating factor (GM-CSF), interleukin 4 (IL-4), and tumor necrosis factor alpha (TNF- $\alpha$ ), cytokines known to be pivotal in vivo and in vitro for DC generation from monocytes or $\mathrm{CD} 4^{+}$stem cells. As assessed by FACS analysis, MUTZ- 3 cells cultured for $7 \mathrm{~d}$ in the presence of cytokine cocktails developed into irregularly shaped-DC (see below), as characterized by the acquisition of CD1a, CD11c, CD40 and CD80 antigens, and showed elevated MHC Class I, Class II and CD86 antigen expressions on the cell surface (Figure 1). Compared to MUTZ-3 cell line, MUTZ3-derived DC (MuDC) showed a slightly enhanced phagocytic activity and reduced expression of IL-3
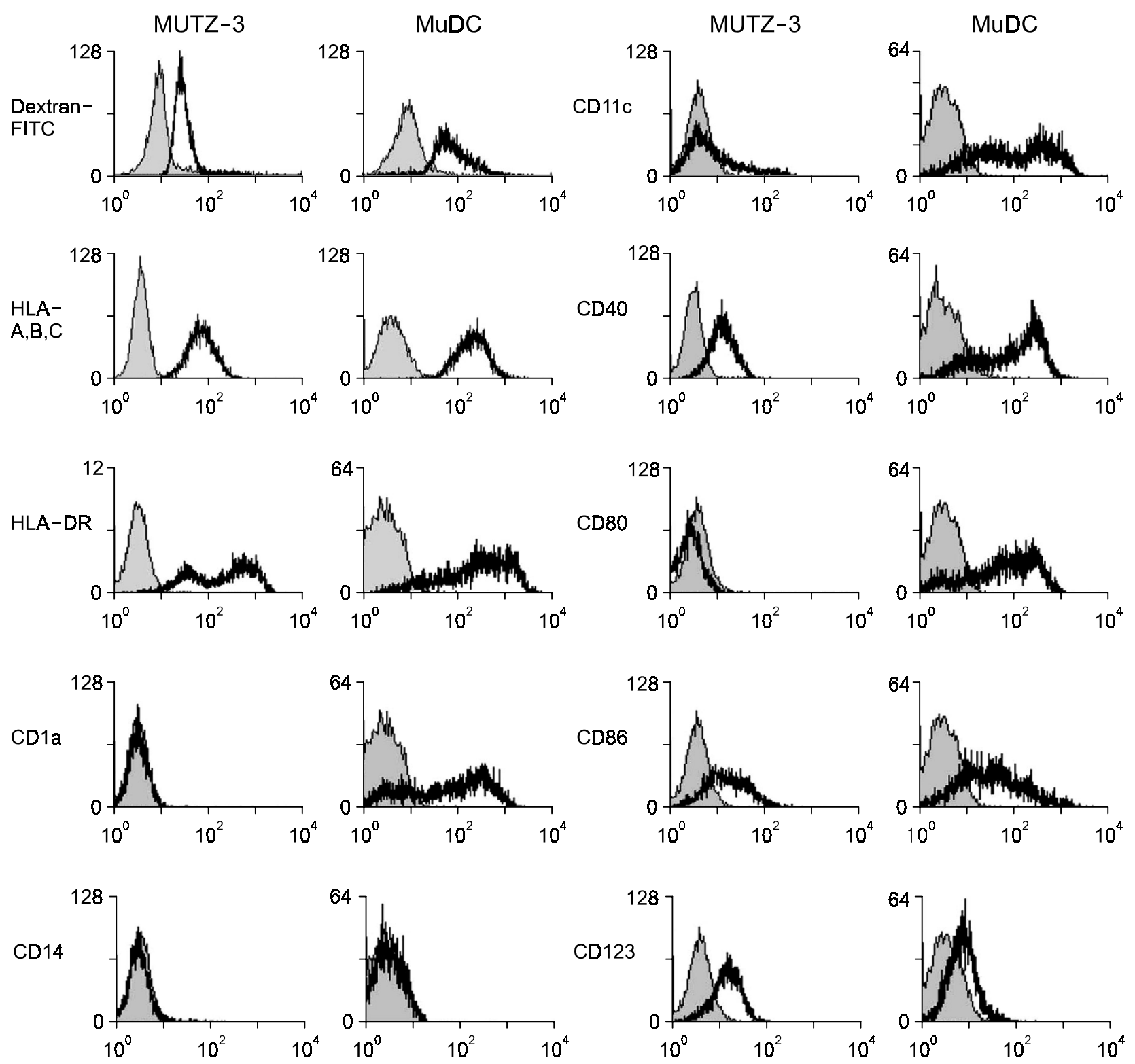

Figure 1. MUTZ-3-derived DC (MuDC) show characteristics of immature DC. Phenotype of surface antigen expression on unstimulated MUTZ-3 cells and MuDC. MUTZ-3 cells were cultured in a 12-well tissue culture plate in the presence of GM-CSF, IL-4 and a low-dose TNF- $\alpha$ for $7 \mathrm{~d}$, as described in Materials and Methods. Cells were stained with PE- or FITC-labeled antigen-specific mAbs. Surface antigen expression was measured using a flow cytometer and analyzed with WinMDI 2.8 (The Scripps Research Institute). Data are from a representative experiment of three independent assays. 
A

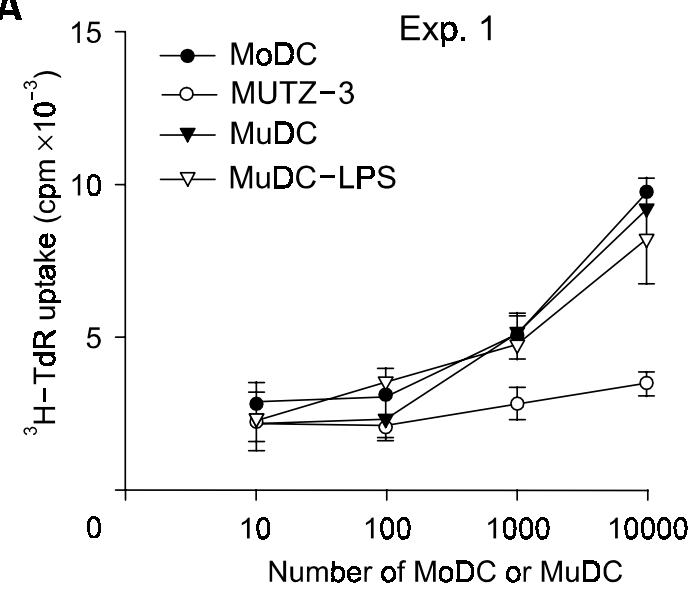

B

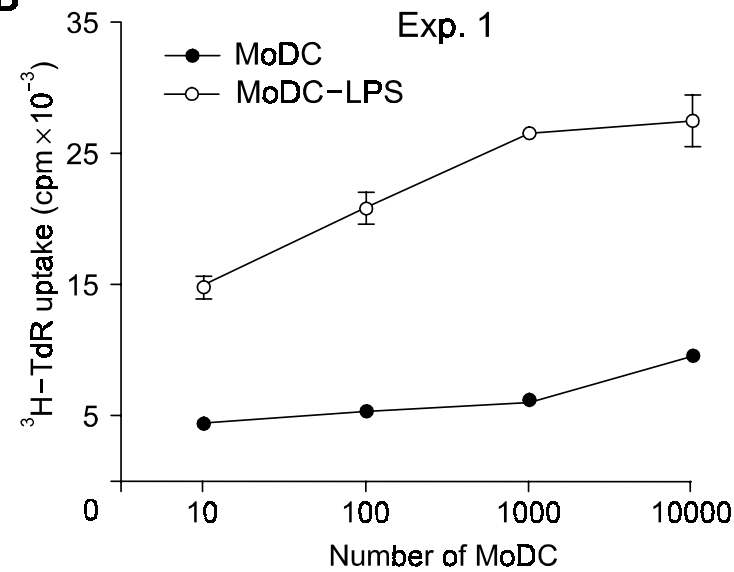

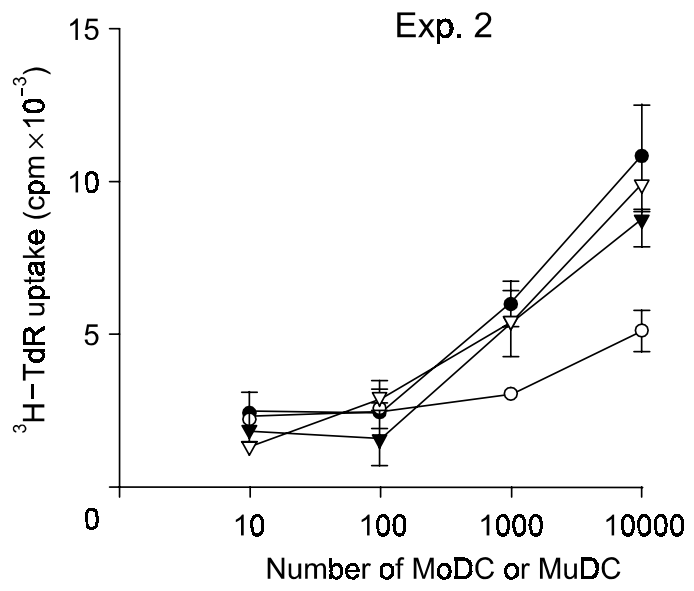

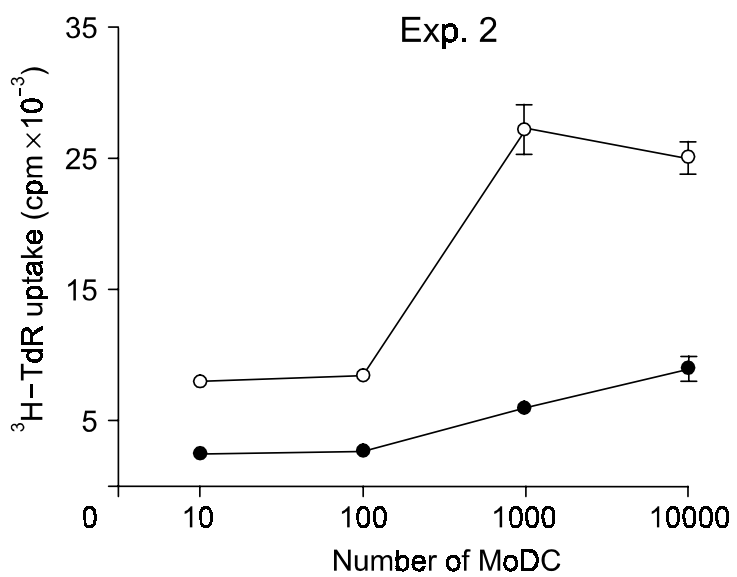

Figure 2. Enhancement of allogeneic T cell proliferation by co-culturing them with MuDC or MoDC. (A) Allogeneic T cells $\left(5 \times 10^{4}\right)$ were incubated with various numbers of irradiated (3000 rad) MUTZ-3 cells, MuDC, or MoDC. Immature MuDC were stimulated with LPS (1 $\mu \mathrm{g} / \mathrm{ml})$ for $24 \mathrm{~h}$ and then co-cultured with allogeneic T cells. The proliferation of T cells was determined in triplicate after $5 \mathrm{~d}$ by pulsing with $1 \mu \mathrm{Ci}$ of $\left[{ }^{3} \mathrm{H}\right]$ thymidine for the last $18 \mathrm{~h}$ and counting with a liquid scintillation counter. (B) MoDC were cultured with allogeneic T cells, with or without LPS pre-treatment. T cell proliferation was determined as described in $(A)$.

receptor protein, CD123. To check the ability of MuDC to activate allogeneic $T$ cells, parent cells or MuDC were treated with purified allogeneic $T$ cells for $5 \mathrm{~d}$ and analyzed for $\mathrm{T}$ cell proliferation. As expected, while parent cells were poor stimulators of primary allogeneic $T$ cell responses, MuDC induced significantly increased $T$ cell proliferation in two sets of experiments, showing a stimulatory activity comparable to that of differentiated MoDC (Figure 2A). However, when MuDC were stimulated with LPS for $24 \mathrm{~h}$ before being co-cultured with $\mathrm{T}$ cells, the $\mathrm{T}$ cell proliferative response was not significantly increased by LPS treatment. In contrast, LPS treatment of MoDC enhanced allostimulatory effect of DC (Figure
2B). Taken together, these results demonstrate that although MUTZ-3 leukemia cells are induced to differentiate into MuDC, which show enhanced allostimulatory activity, MuDC treated with LPS do not produce higher levels of allogeneic $\mathrm{T}$ cell stimulation than non-treated MuDC.

\section{Induction of distinct MuDC maturation by TNF- $\alpha$, CD40 or LPS stimulation}

Proinflammatory cytokines such as TNF- $\alpha$ and IL-1 $\beta$ also trigger maturation, as does LPS or the ligation of CD40, a TNF receptor family member abundant on DC. Thus, we determined the response of MuDC 

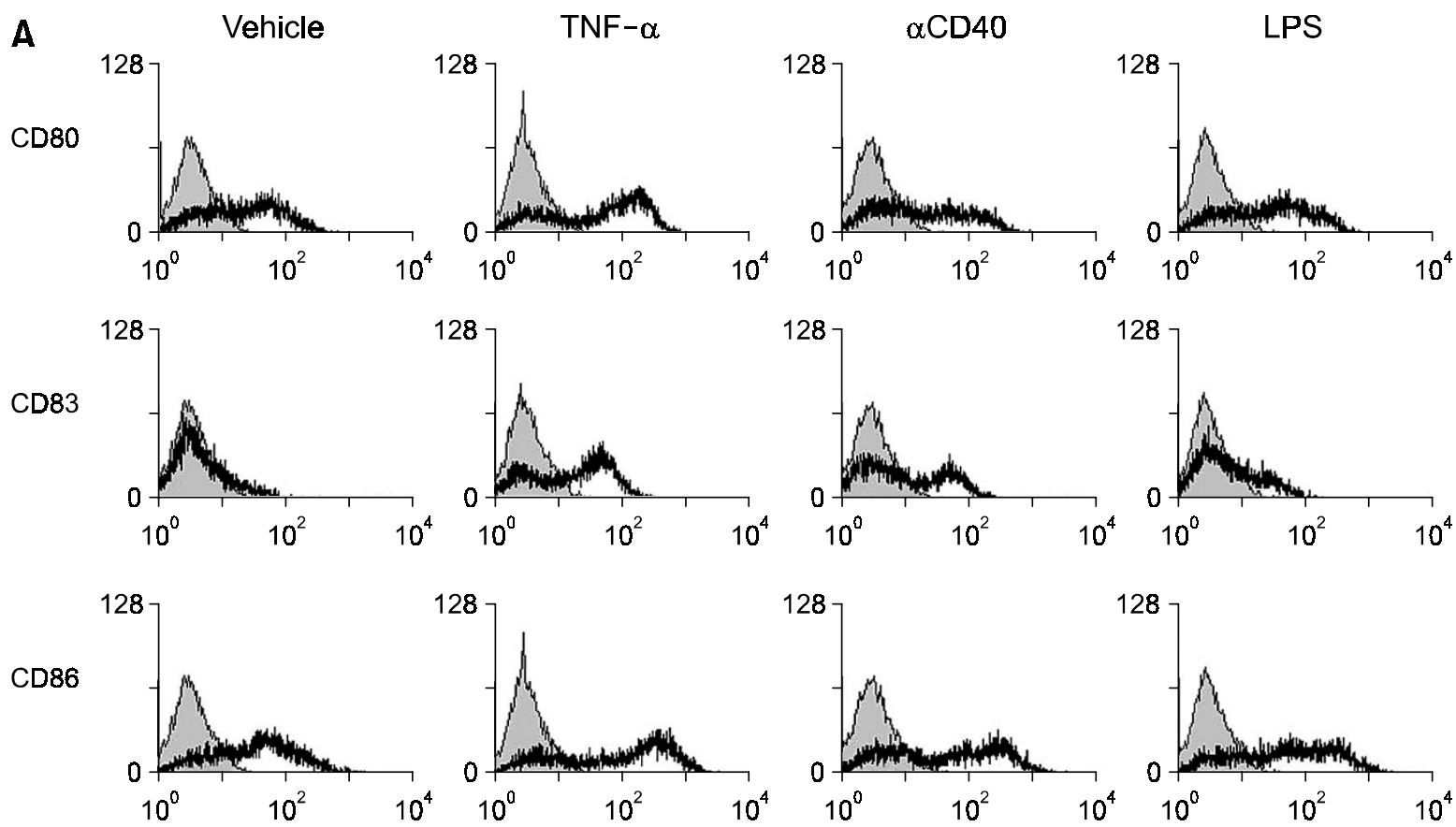

B

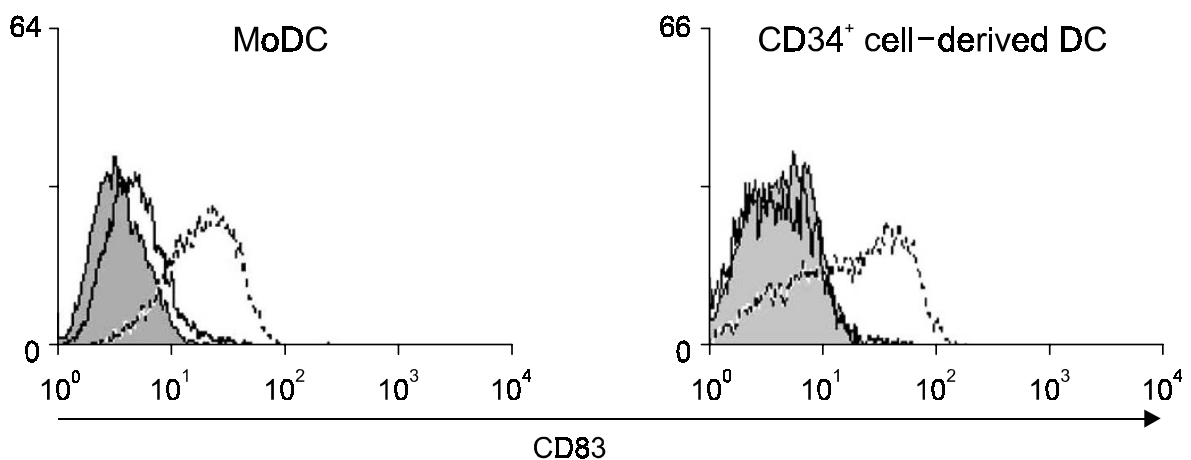

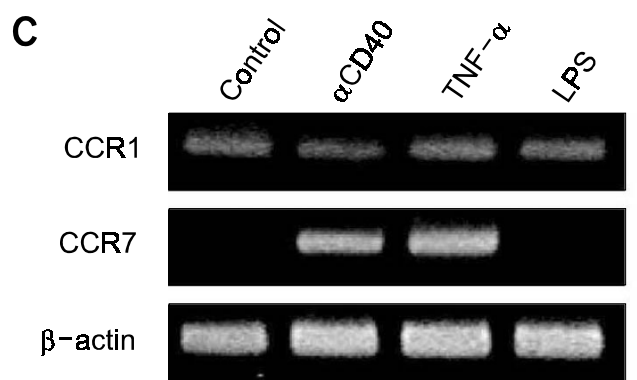

MuDC

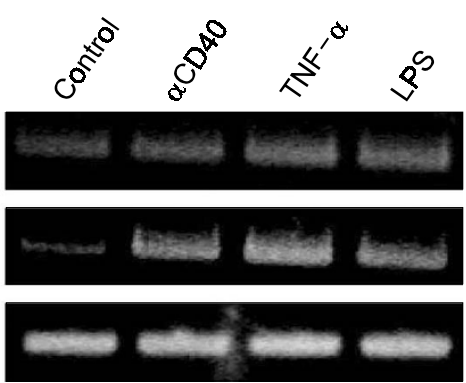

MoDC

Figure 3. LPS stimulation of MuDC does not induce the surface antigen expression of the mature phenotype. (A) MuDC were stimulated with agonistic anti-CD40 Ab (G28.5) $(1 \mu \mathrm{g} / \mathrm{ml})$, of TNF- $\alpha(200 \mathrm{ng} / \mathrm{ml})$, or LPS $(1 \mu \mathrm{g} / \mathrm{ml})$ for $24 \mathrm{~h}$. Cells were harvested and stained with PE- or FITC-labeled antigen-specific mAbs and antigen expression was measured using a flow cytometer. Shaded histograms show results obtained using irrelevant isotype-matched fluorescence-labeled Ig. (B) LPS stimulation of monocyte- or CD34-positive cord blood-derived DC induced CD83 expression. Cells were stained with PE-labeled CD83-specific mAb (dotted line). The shaded histogram indicates unstained cells and the open histogram represents cells stained with isotype control antibody. (C) Expression of CCR1 or CCR7 mRNA in stimulated MuDC or MoDC. Cells were stimulated with anti-CD40 antibody $(1 \mu \mathrm{g} / \mathrm{ml})$, TNF- $\alpha(200 \mathrm{ng} / \mathrm{ml})$ or LPS $(1 \mu \mathrm{g} / \mathrm{ml})$ and total RNAs were isolated from control or stimulated cells. CDNAs were synthesized and amplified as described in Materials and Methods. An aliquot of the reaction products obtained was electrophoresed on an ethidium bromide-stained $1.5 \%$ agarose gel. The figure is representative of three independent experiments. 
to TNF- $\alpha$, CD40 or LPS stimulation. As shown in Figure 3A, TNF- $\alpha(200 \mathrm{ng} / \mathrm{ml})$ treatment for $24 \mathrm{~h}$ upregulated the expressions of CD80 and CD86 and induced CD83 expression. The stimulation of MuDC with anti-CD40 mAb also induced the upregulation of the costimulatory antigens and the neoexpression of CD83. In contrast, LPS did not induce changes in these antigen expressions on MuDC. The MUTZ-3 cell line did not respond to these kinds of stimulation, showing no changes in cell surface antigen expression (data not shown). To determine whether the lack of stimulation by LPS is due to a defect of LPS itself, MoDC and $\mathrm{CD}_{3} 4^{+}$cell-derived DC were stimulated with LPS for $24 \mathrm{~h}$. LPS was found to promote the maturation of MoDC and CD34 ${ }^{+}$ cell-derived DC, as confirmed by the induction of CD83 expression (Figure 3B), indicating LPS is functional in this context. We next tested whether resistance to the maturation-inducing effect of LPS in MuDC is repeated in chemokine receptor expression on cell surface of MuDC. The expression of CCR7 in MuDC, as determined by RT-PCR, was significantly induced by treatment with anti-CD40 $\mathrm{mAb}$ or TNF- $\alpha$, but not with LPS (Figure $3 \mathrm{C}$ ). The level of CCR1 expression in control MuDC was low, and thus the down-regulation of CCR 1 expression by stimuli was less clear. In the case of MoDC, stimulation with anti-CD40 mAb, TNF- $\alpha$, or LPS induced the upregulation of CCR7. Together these results indicate that the maturation program, which follows CD40 or TNF- $\alpha$ stimulation, is operating in MuDC, but that after LPS exposure is defective.

\section{Cytokine production after the stimulation of MuDC by LPS or CD40 ligation}

In an attempt to verify cytokine production in stimulated MuDC, we investigated whether the production of $\mathrm{IL}-12$ or $\mathrm{IL}-10$ is triggered by LPS stimulation or CD40 ligation. As shown in Figure 4, MoDC before exposure to LPS were not able to produce IL-12 and IL-10, whereas after LPS exposure they produced high levels of IL-12 and IL-10. CD40 stimulation also resulted in a significant amount of IL-12 production in MoDC, as detected by ELISA using the supernatant from cultured cells (Figure 4B). However, IL-10 production was not detected after CD40 stimulation of MoDC (Figure 4C). By contrast, although CD40, but not LPS, stimulation of MuDC induced formation of cell cluster representing maturation phenotype (Figure 4A), LPS or CD40 stimulation of MuDC for $24 \mathrm{~h}$ did not lead to
A

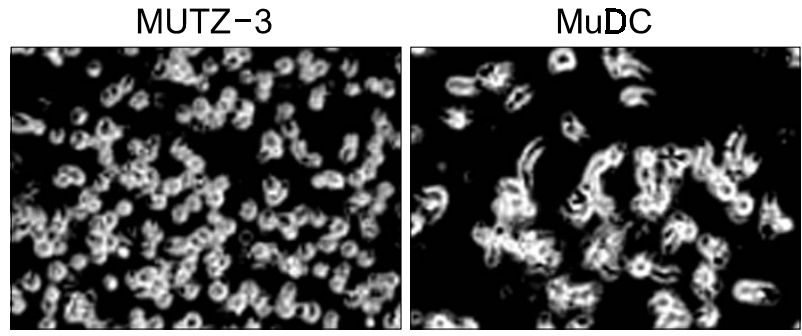

B

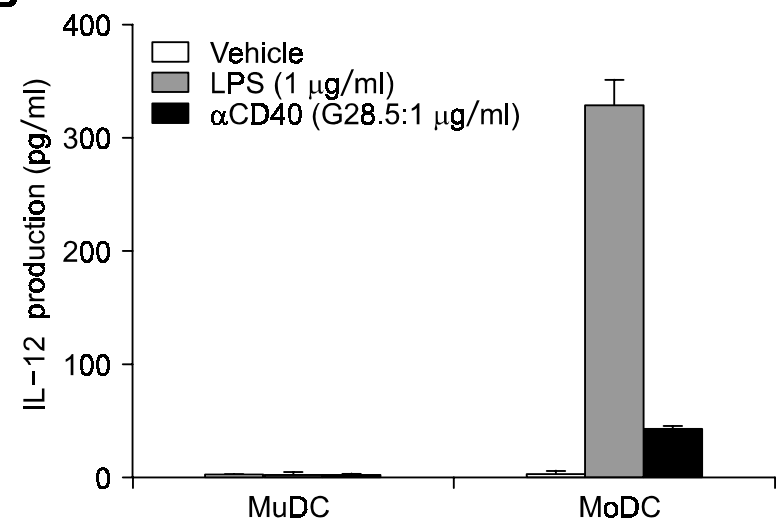

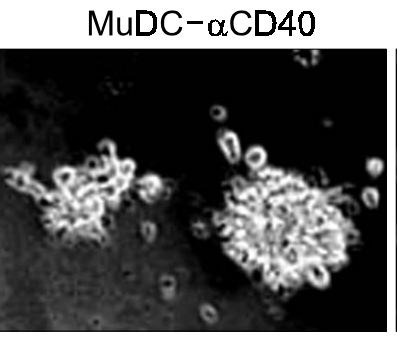

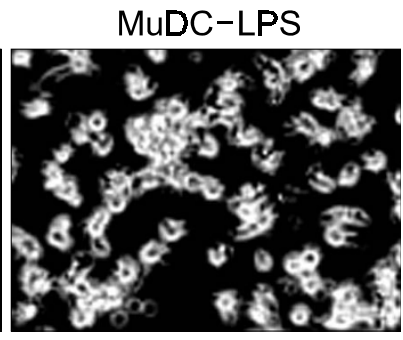

C

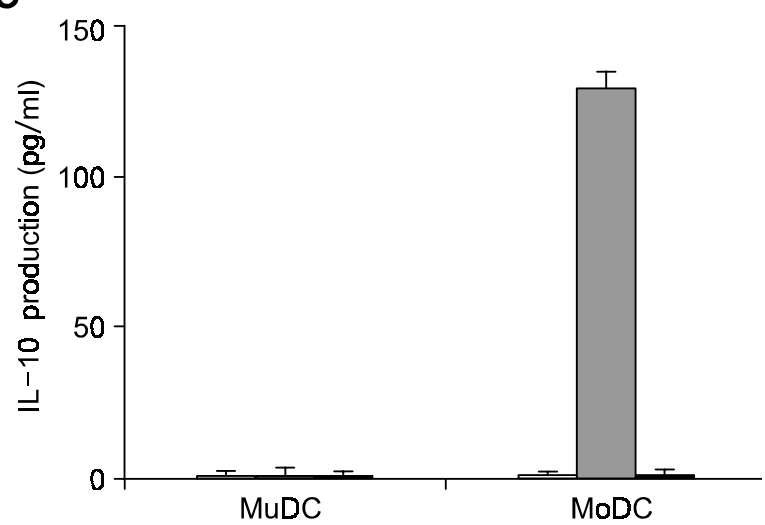

Figure 4. LPS do not induce cytokine production in MuDC. (A) After $7 \mathrm{~d}$ of culture to induce differentiation, MuDC and MoDC were washed twice and stimulated with LPS $(1 \mu \mathrm{g} / \mathrm{ml})$ or agonistic anti-CD40 Ab (1 $\mu \mathrm{g} / \mathrm{ml})$ for $24 \mathrm{~h}$. (B, C) Culture supernatants were collected and IL-12 p70 or IL-10 production induced by unstimulated or stimulated DC was measured by ELISA. 
A
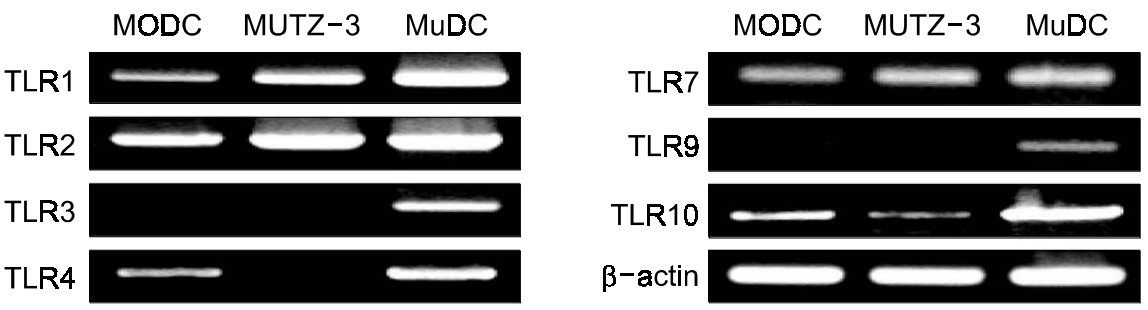

B
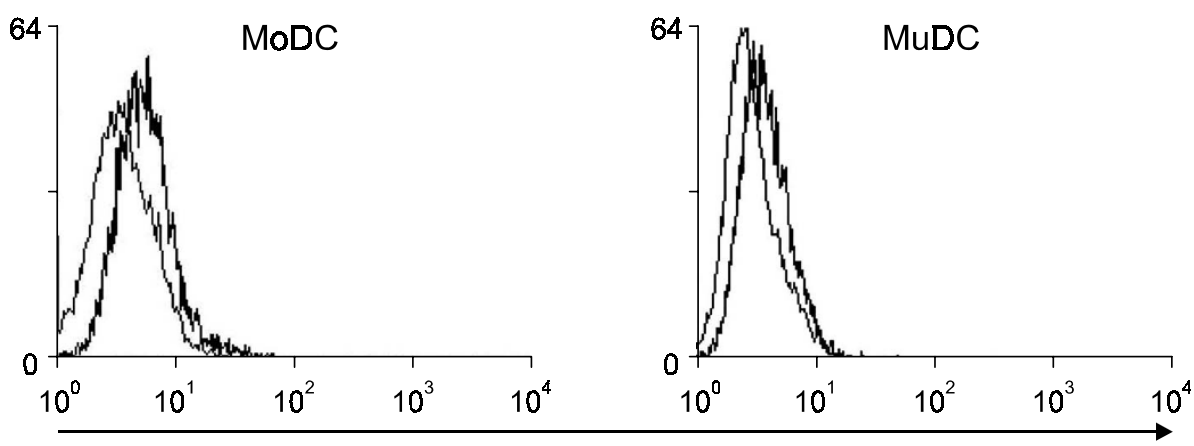

TLR4

Figure 5. TLR4 expression in MoDC and MuDC. (A) The expressions of TLR1, 2, 3, 4, 7, 9 and TLR10 mRNAs in MoDC or MuDC were analyzed by RT-PCR. It was performed as described in Materials and Methods. The figure shown is representative of three independent experiments. (B) Analysis of the surface expressions of TLR4 in MoDC and MuDC. MuDC or MoDC were washed twice and stained with anti-TLR4 antibody for 30 min on ice, followed by goat $F\left(a b^{\prime}\right)_{2}$ anti-mouse Ig's-FITC conjugate as a secondary antibody (bold line). Control staining was done with a secondary antibody only (thin line). TLR4 expression was measured by flow cytometry and analyzed with WinMDI 2.8. The data shown represent the results of two experiments.

the production of IL-12 or IL-10, again indicating that LPS did not deliver the signal that critically affects cytokine production in MuDC.

\section{TLR4 mRNA and protein expression in MuDC}

Since TLR4, a central component for recognition of Gram-negative LPS, plays a crucial role in LPS signaling to induce DC maturation, we wondered whether the unresponsiveness of MuDC to LPS could be due to the defective expression of TLR4 in MuDC. In addition, it has been shown that TLR2 functions as a receptor for atypical LPS produced by Leptospira interogans (Werts et al., 2001) and Porphyromonas gingivitis (Hirschfeld et al., 2001). When we analyzed a number of TLR mRNAs in both MoDC and MuDC by RT-PCR, we found that TLR2 and TLR4 were expressed at comparable levels (Figure 5A). Moreover, when the surface expression of TLR4 was examined by flow cytometry using TLR4-specific monoclonal antibody, TLR4 expression on cell surface was detected in both MoDC and MuDC (Figure 5B). Our results therefore suggest that the lack of LPS responsiveness of MuDC to LPS is not the result of the differential expression of TLR4.
TNF- $\alpha$ or IL- $1 \beta$, but not LPS, induces p38 mitogen-activated protein kinase and ERKs activation in MuDC

Signals that promote DC maturation and survival may follow different pathways. It has been previously shown that the LPS-induced maturation of DC is also mediated by the activation of p38-MAPK (Arrighi et al., 2001), and that LPS induces early ERKs activation, which appears to be required for DC survival (Rescigno et al., 1998; Bouchon et al., 2001). Therefore, we investigated whether LPS or TNF- $\alpha$ differentially controls MAPK and ERKs activation in MUTZ-3-derived DC. Stimulation with $200 \mathrm{ng} / \mathrm{ml}$ of TNF- $\alpha$ induced the phosphorylation of MAPK (Figure 6A). The maximal phosphorylations of p38 and ERK1/2 were observed to reach a maximum between 5 and 10 min after TNF- $\alpha$ stimulation, and to decrease after $30 \mathrm{~min}$. LPS stimulation was retarded by comparison and showed significantly lower peak p38 MAPK phosphorylation, and barely detectable ERK1 and ERK2 phosphorylation. Since the LPS signaling cascades are known to be similar in some respects to the down-stream cascades mediated by IL-1 receptor (IL-1R), we tested whether IL-1 $\beta$ could transduce signals that 
A

\begin{tabular}{|c|c|c|c|c|c|c|c|c|c|c|}
\hline \multirow{2}{*}{\multicolumn{2}{|c|}{0}} & \multicolumn{4}{|c|}{ LPS } & \multicolumn{4}{|c|}{ TNF $-\alpha$} & \\
\hline & & 5 & 10 & 30 & 60 & 5 & 10 & 30 & 60 & \\
\hline Phospho-p38-MAPK & 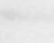 & 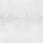 & $=$ & - & $=$ & - & 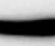 & - & - & \\
\hline
\end{tabular}

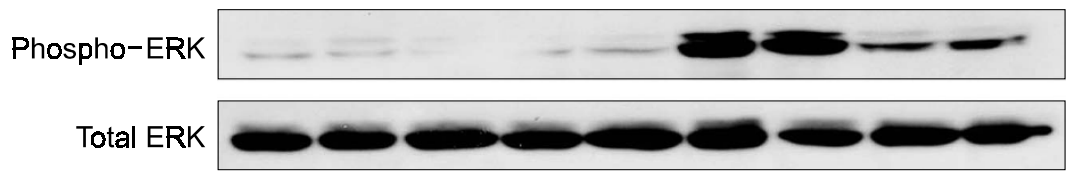

IL-1 $\beta$

B

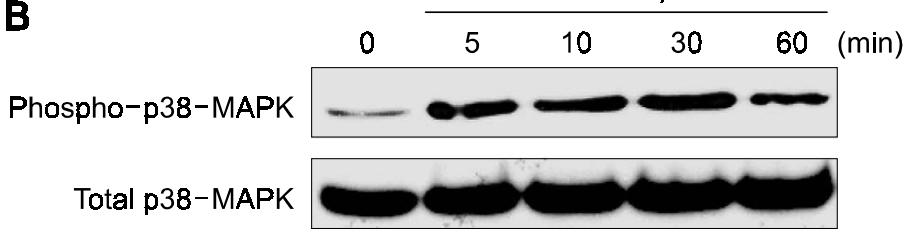

Phospho-ERK - - - -
Total ERK

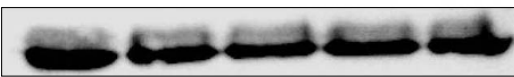

Figure 6. Activations of p38-MAPK and ERK in MuDC after stimulation with LPS, TNF- $\alpha$ or IL-1 $\beta$. MuDC were stimulated with either LPS $(1 \mu \mathrm{g} / \mathrm{ml})$, TNF- $\alpha(200 \mathrm{ng} / \mathrm{ml})(\mathrm{A})$ or IL-1 $\beta(20$ $\mathrm{ng} / \mathrm{ml})(B)$ for the indicated times. The activations of p38-MAPK and ERK were assessed as described in Materials and Methods. Immunoblotting using antibodies against total p38-MAPK and ERK was done as a loading control. activate MAPK. The treatment of MuDC with IL-1 $\beta$ was found to induce maximal p38 and ERK1/2 phosphorylations between 10 and $30 \mathrm{~min}$ (Figure $6 \mathrm{~B})$. These data indicate that TNF- $\alpha$ or IL-1 $\beta$, but not LPS, induces the early phosphorylation of ERK $1 / 2$ and p38 MAPK in MuDC, and that signaling pathways incorporating LPS and IL-1 $\beta$ are fully functional in MuDC.

\section{Co-culture with MoDC, but not with MuDC, induces NK cell activation}

To extend our study to NK cell activation induced during co-culture with DC stimulated by LPS, we co-cultured MoDC or MuDC with purified allogeneic NK cells in the presence or absence of LPS for $24 \mathrm{~h}$. Primary NK cells themselves showed moderate cytolytic activity against K562 target cells, but after co-culture with unstimulated MoDC, the cytolytic activity of NK cells was significantly enhanced (Figure 7A). The addition of LPS to a co-culture of NK cells with MoDC increased the cytotoxic effect of NK cells. However, this stimulatory effect was not observed when MoDC were replaced with MuDC, even in the presence of LPS, because the cytotoxicity of NK cells was not significantly enhanced after co-culture with MuDC. IFN- $\gamma$ production was clearly more pronounced after co-culture with LPS-stimulated MoDC, and was not detected in NK cells alone or in NK cells co-cultured with MuDC (Figure 7B), suggesting that IFN- $\gamma$ was not produced in allogeneic MoDC or MuDC. Additionally IL-12 was not produced in MuDC, when they were co-cultured with NK cells in the presence of LPS (Figure 7C). Altogether, these results indicate that MuDC might be defective in their ability to stimulate innate immune responses mediated via NK cells, and especially in terms of the ability to sense microbial products like LPS.

\section{Discussion}

In the present study, we demonstrate that a myeloid leukemia cell line can be induced in vitro to differentiate into fully functional cells with antigen presenting activity and DC features by short-term culture with a combination of cytokines. We showed as do MoDC, leukemic DC elicit significant $T$ cell proliferation in allo-MLR. Importantly, the signaling generated by LPS, which is a potent inducer of human DC maturation and survival, did not lead to the maturation of leukemic DC. LPS treatment of leukemic DC did not trigger the activation pathway, as detected by the phosphorylated products of p38-MAPK and ERK. Defective LPS responsiveness therefore resulted in the inability of DC to stimulate primary NK cells in DC-NK cell co-culture. 
A

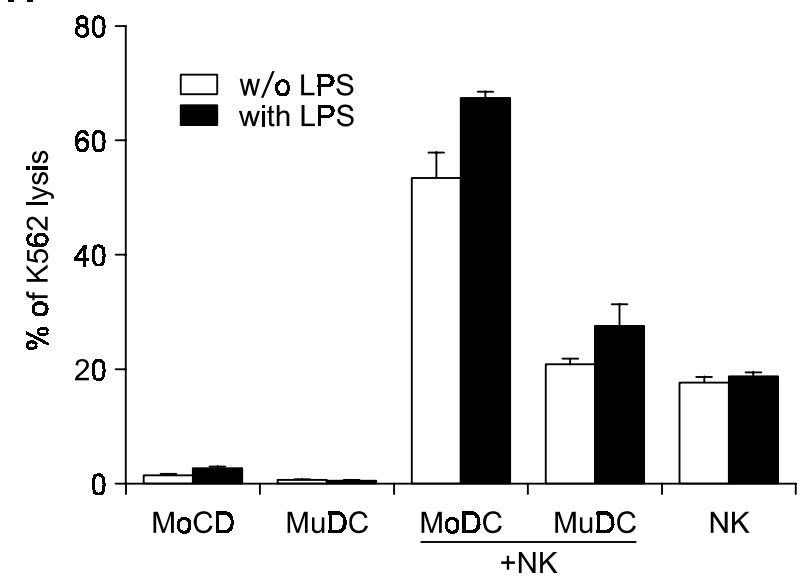

C

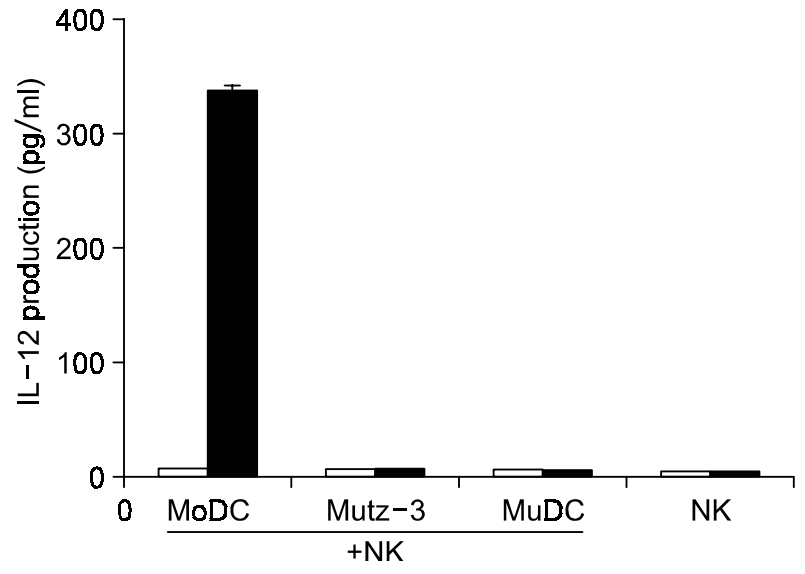

Proinflammatory cytokines, such as TNF- $\alpha$ and $\mathrm{IL}-1 \beta$ also trigger maturation, as does the ligation of CD40, a TNF receptor family member abundant on DC. In our leukemic DC, DC maturation, as detected by the enhancement of surface antigen expression and CCR7 expression, was induced by TNF- $\alpha$ or agonistic CD40 mAb. These findings indicate that the maturation process of leukemic DC can be initiated by a variety of inflammatory stimuli. The increased expression of costimulatory molecules like CD80 and CD86 has been correlated with the capacity of DC to induce naive $\mathrm{CD4}^{+} \mathrm{T}$ cell proliferation, which is associated with the expression of CD83 (Chen et al., 1998). In contrast, in the present study, no difference was observed between the untreated control and LPS-treated leukemic DC in terms of the phenotypes of DC maturation. The signaling subunit of the LPS receptor in DC is TLR4, a member of TLR family. This is a type 1 transmembrane protein, which contains in its cytoplasmic region a Toll-interleukin-1 receptor domain that links the receptor to the signaling machinery shared by IL-1 and IL-18 (Akira et al., 2001). When TLR4
B

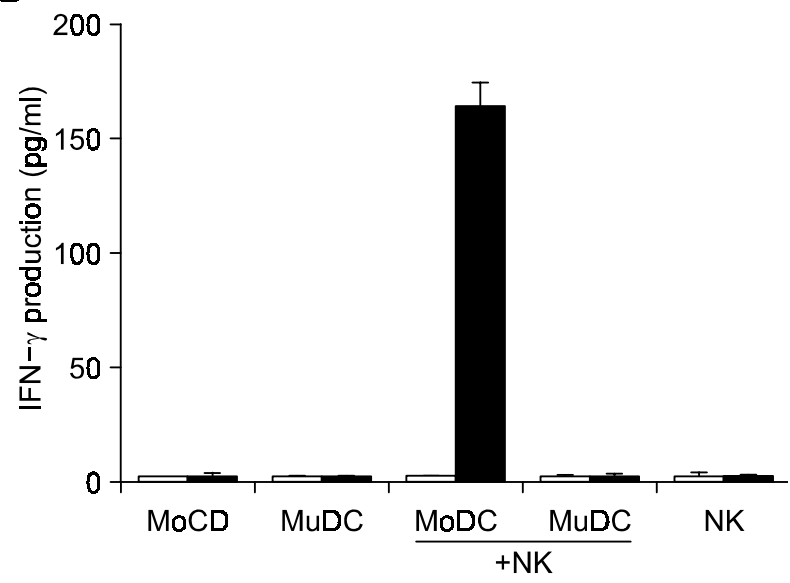

Figure 7. Cytolytic activity and IFN- $\gamma$ production by NK cells in mixed culture of NK cells with either MuDC or MoDC. Fresh NK cells and MuDC or MoDC were co-cultured in the absence or presence of LPS $(1 \mu \mathrm{g} / \mathrm{ml})$ for $24 \mathrm{~h}$. After co-culturing, the cytolytic activities of the NK cells were evaluated using ${ }^{51} \mathrm{Cr}$-labeled K562 cell line as target cells (A). Supernatants were collected to evaluate IFN- $\gamma(\mathrm{B})$ and IL-12 production (C). Cytokine concentration was measured as described in Materials and Methods. The data shown represent one of the two individual experiments.

expression in leukemic DC and in MoDC was quantified by RT-PCR and flow cytometric analysis, comparable levels of TLR4 mRNA and protein were found, indicating that the defective response of leukemic DC to LPS was not due to the level of TLR expression. In addition, TLRs are found to respond to different microbial products, and the signaling pathways activated are very similar to those generated by the proinflammatory cytokine IL-1. It was therefore remarkable that on IL-1 stimulation, p38MAPK and ERK were rapidly activated in leukemic DC. It is known that TLR proteins recognize mycobacterial products and activate signaling pathways, some of which are shared with the IL-1 receptor family, leading to NF- $\kappa B$ signal transduction and the production of proinflammatory cytokines (Hoffmann et al., 1999). Our data, therefore, suggest that signaling mediators important for the downstream signal transduction are functionally intact. However, there is an increasing body of evidence indicating that LPS tolerance may be induced under conditions whereby cells express comparable levels of TLR4, MD-2, MyD88, and TRAF-4 protein (Medvedev et 
al., 2000; Medvedev et al., 2001). For example, LPS-tolerant cells were found to exhibit a markedly impaired capacity to recruit MyD88 to TLR4 in response to LPS (Medvedev et al., 2002). There also exist MAL/TIRAP-dependent pathways unique to TLR4 (O'Neill, 2002). Although the expression of MAL/TIRAP in our leukemic DC was confirmed by RT-PCR analysis (data not shown), it remains to be resolved whether MAL/TIRAP is functionally defective.

Recently, it was reported that common, cosegregating missense mutations (Asp299Gly and Thr399lle) affecting the extracellular domain of the TLR4 receptor are associated with a blunted response to inhaled LPS in humans (Arbour et al., 2000). Based on this report, we hypothesized that this mutation in the TLR4 of our leukemic DC may be associated with a diminished responsiveness to LPS treatment. However, we were not able to find a substitution of Asp by Gly at nt $896(A \longrightarrow T)$ or a substitution of Thr by lle at nt $1196(\mathrm{C} \longrightarrow \mathrm{T})$ at TLR4 cDNA by direct sequencing analysis (data not shown). In addition, we found that the nucleotide sequences encoding the extracellular domain of TLR4 do not have changes. It is therefore possible that the intracellular domain of TLR4 or its interaction with adaptor proteins in the cytoplasm is impaired. In fact, it has been previously reported that, although changes in gene sequence alter the ability of the host to respond to environmental stress, not all subjects hyporesponsive to LPS have mutations in TLR4, and that not all with the TLR4 mutation are hyporesponsive to inhaled LPS (Arbour et al., 2000).

The availability of leukemia-derived DC and their capacity to enhance tumor recognition is a promising approach to immunotherapy in AML, and hopefully will pave the way for therapeutic options in other hematological malignancies (Charbonnier et al., 1999; Choudhury et al., 1999). However, in AML and in other leukemias, the generation of potent tumorspecific cytotoxic effectors capable of tumor control may be subsequently counterbalanced by a variety of mechanisms leading to anergy (Mohty et al., 2002b). For example, patients with AML, as compared to healthy individuals, may have in vivo a dramatic quantitative imbalance in their circulating blood DC subsets, with a great number of patients showing a tremendous expansion of either myeloid dendritic cells (MDC), plamacytoid dendritic cells (PDC) or both subsets (Mohty et al., 2001). In addition, stimulation via the CD40 pathway enhanced the secretion of IL-12p70 by CD14 ${ }^{+}$cellderived leukemic DC and circulating MDC precursorderived $D C$, but there was a trend towards a higher level of secretion of $\mathrm{IL}-10$ by $\mathrm{DC}$ derived from leukemic circulating MDC (Mohty et al., 2002a). These leukemic DC may not help in the generation of anti-leukemic cytotoxic CD8 ${ }^{+} \mathrm{T}$ cells. Moreover, in chronic myeloid leukemia $(\mathrm{CML})$, the maturation of leukemic MoDC triggered by a natural stimulus like LPS, is abnormal and may be caused by an aberrant TNF- $\alpha$ response in these cells (Eisendle et al., 2003). Other results show that DC generated from the blood of CML patients display decreased T-cell stimulatory activity in vitro when tested with allogeneic responder T cells (Wang et al., 1999). Thus, the design of a clinical DC-based vaccine immunotherapy protocol requires a concise functional characterization of $D C$ as well as reflection on the crucial role of the route and timing of vaccine delivery to ensure potent specific cytotoxic effectors and helper T lymphocytes.

Taken together, our results established differences in the functions of myeloid leukemia cell-derived DC as compared to blood MoDC. In particular, the unresponsiveness of leukemic DC to LPS stimulation might have a significant impact on the functions of DC, because LPS is known to induce human DC maturation and survival. In fact, though MoDC stimulated NK cells in the presence of LPS, MuDC could not enhance NK cell activities. TLRs have a central role in the initiation of innate cellular immune responses and in subsequent adaptive immune responses to microbial pathogens, and the interaction between MoDC and NK cells plays important role in initiating innate immune response. Therefore, our data suggest that myeloid leukemia derived DC may have an impaired ability to initiate innate immune response and recognize endogenous ligands that represent "danger signal". The mechanisms underlying the defect in TLR-mediated cell signaling pathway in MuDC are under current investigation.

\section{Acknowledgment}

This work was supported by grants, R11-2005-01703001 of the Research Center for Women's Diseases from the KOSEF, and 05092-CEL-326 from the Korean Food and Drug Administration.

\section{References}

Akira S, Takeda K, Kaisho T. Toll-like receptors: critical proteins linking innate and acquired immunity. Nat Immunol 2001;2:675-80

Arbour NC, Lorenz E, Schutte BC, Zabner J, Kline JN, Jones M, Frees K, Watt JL, Schwartz DA. TLR4 mutations are associated with endotoxin hyporesponsiveness in humans. Nat Genet 2000;25:187-91

Arrighi JF, Rebsamen M, Rousset F, Kindler V, Hauser C. A critical role for p38 mitogen-activated protein kinase in the 
maturation of human blood-derived dendritic cells induced by lipopolysaccharide, TNF-alpha, and contact sensitizers. J Immunol 2001;166:3837-45

Banchereau J, Briere F, Caux C, Davoust J, Lebecque S, Liu YJ, Pulendran B, Palucka K. Immunobiology of dendritic cells. Annu Rev Immunol 2000;18:767-811

Bouchon A, Hernandez-Munain C, Cella M, Colonna M. A DAP12-mediated pathway regulates expression of CC chemokine receptor 7 and maturation of human dendritic cells. J Exp Med 2001;194:1111-22

Charbonnier A, Gaugler B, Sainty D, Lafage-Pochitaloff M, Olive D. Human acute myeloblastic leukemia cells differentiate in vitro into mature dendritic cells and induce the differentiation of cytotoxic $T$ cells against autologous leukemias. Eur J Immunol 1999;29:2567-78

Chen B, Shi Y, Smith JD, Choi D, Geiger JD, Mule JJ. The role of tumor necrosis factor alpha in modulating the quantity of peripheral blood-derived, cytokine-driven human dendritic cells and its role in enhancing the quality of dendritic cell function in presenting soluble antigens to CD4+ T cells in vitro. Blood 1998;91:4652-61

Choi SC, Kim KD, Kim JT, Kim JW, Yoon DY, Choe YK, Chang YS, Paik SG, Lim JS. Expression and regulation of NDRG2 (N-myc downstream regulated gene 2) during the differentiation of dendritic cells. FEBS Lett 2003;553:413-8

Choudhury BA, Liang JC, Thomas EK, Flores-Romo L, Xie QS, Agusala K, Sutaria S, Sinha I, Champlin RE, Claxton DF. Dendritic cells derived in vitro from acute myelogenous leukemia cells stimulate autologous, antileukemic T-cell responses. Blood 1999:93:780-6

Eisendle K, Lang A, Eibl B, Nachbaur D, Glassl H, Fiegl M, Thaler J, Gastl G. Phenotypic and functional deficiencies of leukaemic dendritic cells from patients with chronic myeloid leukaemia. Br J Haematol 2003;120:63-73

Fitzgerald KA, Palsson-McDermott EM, Bowie AG, Jefferies CA, Mansell AS, Brady G, Brint E, Dunne A, Gray P, Harte MT, McMurray D, Smith DE, Sims JE, Bird TA, O'Neill LA. Mal (MyD88-adapter-like) is required for Toll-like receptor-4 signal transduction. Nature 2001;413:78-83

Hirschfeld M, Weis JJ, Toshchakov V, Salkowski CA, Cody MJ, Ward DC, Qureshi N, Michalek SM, Vogel SN. Signaling by toll-like receptor 2 and 4 agonists results in differential gene expression in murine macrophages. Infect Immun 2001;69:1477-82

Hoffmann JA, Kafatos FC, Janeway CA, Ezekowitz RA. Phylogenetic perspectives in innate immunity. Science 1999; 284:1313-8

Horng T, Barton GM, Medzhitov R. TIRAP: an adapter molecule in the Toll signaling pathway. Nat Immunol 2001;2:835-41

Hoshino K, Takeuchi O, Kawai T, Sanjo H, Ogawa T, Takeda Y, Takeda K, Akira S. Cutting edge: Toll-like receptor 4 (TLR4)-deficient mice are hyporesponsive to lipopolysaccharide: evidence for TLR4 as the Lps gene product. J Immunol 1999;162:3749-52

Masterson AJ, Sombroek CC, De Gruijl TD, Graus YM, van der Vliet HJ, Lougheed SM, van den Eertwegh AJ, Pinedo HM, Scheper RJ. MUTZ-3, a human cell line model for the cytokine-induced differentiation of dendritic cells from CD34 precursors. Blood 2002;100:701-3

Medvedev AE, Kopydlowski KM, Vogel SN. Inhibition of lipopolysaccharide-induced signal transduction in endotoxintolerized mouse macrophages: dysregulation of cytokine, chemokine, and toll-like receptor 2 and 4 gene expression. $\mathrm{J}$ Immunol 2000;164:5564-74

Medvedev AE, Henneke P, Schromm A, Lien E, Ingalls R, Fenton MJ, Golenbock DT, Vogel SN. Induction of tolerance to lipopolysaccharide and mycobacterial components in Chinese hamster ovary/CD14 cells is not affected by overexpression of Toll-like receptors 2 or 4 . J Immunol 2001;167:2257-67

Medvedev AE, Lentschat A, Wahl LM, Golenbock DT, Vogel SN. Dysregulation of LPS-induced Toll-like receptor 4MyD88 complex formation and IL-1 receptor-associated kinase 1 activation in endotoxin-tolerant cells. J Immunol 2002;169:5209-16

Mohty M, Jarrossay D, Lafage-Pochitaloff M, Zandotti C, Briere $F$, de Lamballeri $X N$, Isnardon D, Sainty D, Olive D, Gaugler B. Circulating blood dendritic cells from myeloid leukemia patients display quantitative and cytogenetic abnormalities as well as functional impairment. Blood 2001; 98:3750-6

Mohty $M$, Isnardon D, Blaise $D$, Mozziconacci MJ, LafagePochitaloff M, Briere F, Gastaut JA, Olive D, Gaugler B. Identification of precursors of leukemic dendritic cells differentiated from patients with acute myeloid leukemia. Leukemia 2002a;16:2267-74

Mohty M, Olive D, Gaugler B. Leukemic dendritic cells: potential for therapy and insights towards immune escape by leukemic blasts. Leukemia 2002b;16:2197-204

O'Neill LA. Toll-like receptor signal transduction and the tailoring of innate immunity: a role for Mal? Trends Immunol 2002;23:296-300

Poltorak A, He X, Smirnova I, Liu MY, Van Huffel C, Du X, Birdwell D, Alejos E, Silva M, Galanos C, et al. Defective LPS signaling in $\mathrm{C} 3 \mathrm{H} / \mathrm{HeJ}$ and $\mathrm{C} 57 \mathrm{BL} / 10 \mathrm{ScCr}$ mice: mutations in TIr4 gene. Science 1998;282:2085-8

$\mathrm{Re} F$, Strominger JL. Heterogeneity of TLR-induced responses in dendritic cells: from innate to adaptive immunity. Immunobiology 2004;209:191-8

Rescigno M, Martino M, Sutherland CL, Gold MR, RicciardiCastagnoli P. Dendritic cell survival and maturation are regulated by different signaling pathways. J Exp Med 1998;188:2175-80

Rescigno M, Borrow P. The host-pathogen interaction: new themes from dendritic cell biology. Cell 2001;106:267-70

Sallusto F, Lanzavecchia A. Efficient presentation of soluble antigen by cultured human dendritic cells is maintained by granulocyte/macrophage colony-stimulating factor plus interleukin 4 and downregulated by tumor necrosis factor alpha. J Exp Med 1994;179:1109-18

Shimazu R, Akashi S, Ogata H, Nagai Y, Fukudome K, 
Miyake K, Kimoto M. MD-2, a molecule that confers lipopolysaccharide responsiveness on Toll-like receptor $4 . \mathrm{J}$ Exp Med 1999;189:1777-82

Suzuki N, Suzuki S, Yeh WC. IRAK-4 as the central TIR signaling mediator in innate immunity. Trends Immunol 2002;23:503-6

Wang C, Al-Omar HM, Radvanyi L, Banerjee A, Bouman D, Squire J, Messner HA. Clonal heterogeneity of dendritic cells derived from patients with chronic myeloid leukemia and enhancement of their T-cells stimulatory activity by IFNalpha. Exp Hematol 1999;27:1176-84
Werts C, Tapping RI, Mathison JC, Chuang TH, Kravchenko V, Saint Girons I, Haake DA, Godowski PJ, Hayashi F, Ozinsky A, Underhill DM, Kirschning CJ, Wagner H, Aderem A, Tobias PS, Ulevitch RJ. Leptospiral lipopolysaccharide activates cells through a TLR2-dependent mechanism. Nat Immunol 2001;2:346-52

Zhang D, Zhang G., Hayden MS, Greenblatt MB, Bussey C, Flavell RA, Ghosh S. A toll-like receptor that prevents infection by uropathogenic bacteria. Science 2004;303: 1522-6 